\title{
A Novel Adaptive Steganographic Technique using Kohonen Neural Network based on Integer Wavelet Transform
}

\author{
Liya Lesly \\ Department of Electronics and communication \\ Federal Institute of Science and Technology
}

(FISAT)

\author{
Rajesh Cherian Roy \\ Department of Electronics and communication \\ Federal Institute of Science and Technology
}

(FISAT)

\begin{abstract}
Steganography allows us to hide a desired confidential information in a covered or host image while image encryption is decomposing the desired information to a nonreadable, non-comprehended manner. Steganography gained importance in the past few years due to the increasing need for providing secrecy in an open environment like the internet. This is made possible by hiding the very existence of the message and make communication undetectable. Steganography is the science of secret message delivery using cover media and has many technical challenges such as high hiding capacity and security. In this paper, a method is proposed to optimize these two requirements by suggesting a novel technique for hiding data in digital images. For this, an adaptive hiding capacity function along with a Kohonen neural network has been employed. This method hides secret data in Integer wavelet coefficients. The Optimal pixel adjustment (OPA) algorithm is applied after embedding the message. A new high capacity image steganographic method based on Kohonen neural network is also introduced. Kohonen network is trained according to the absolute contrast sensitivity of pixels present in cover image. On the receiving side, the original image is not needed for extracting the embedded data. It is observed that the capacity and security is increased with acceptable PSNR and hiding capacity.
\end{abstract}

\section{Keywords}

Steganography, Integer Wavelet Transform, Discrete Cosine Transform, adaptive algorithm, Kohonen NN, Optimal Pixel Adjustment Algorithm.

\section{INTRODUCTION}

Information hiding is a method of thrashing secret data into a host medium so that the hidden data are imperceptible but known to the intended recipient [1]. This can be achieved by concealing the existence of information within seemingly harmless carrier or cover. Steganography means "covered writing" and it [2] concerns itself with ways of embedding a secret message into a cover object, without altering the properties of the cover object evidently. The embedding procedure is typically related with a key, usually a stego-key. Without knowledge of this key it will be difficult for a third party to extract the message or even detect its existence. Once the cover object has data embedded in it, it is called stego object. Steganography differs from cryptography in the sense that it tries to hide the message instead of transforming it so as to obscure its meaning [3]. The host medium may be a digital image, audio, video or any other type of media. Among the different kinds of media, the digital image is most popularly used as the host media to convey secret information. This is due to the fact that they are easy to obtain with reasonable hiding capacity and distortion tolerance [4].
The data hiding schemes can be categorized in two groups: spatial and frequency domain. The first technique is based on embedding message in the Least Significant Bits (LSB) of image pixels. The basic LSB method has a simple implementation and high hiding capacity [5]. However, it has low robustness against some attacks such as low-pass filtering and compression [6]. An alternate method of LSB substitution is OPAP (Optimal Pixel Adjustment Process) [4] in which the image quality of the stego-image can be enhanced sufficiently. In the second method, frequency coefficients of images are selected and then embeds the messages within them.

Different transforms are available for data hiding, such as Discrete Fourier Transform (DFT), the Discrete Wavelet Transform (DWT), Integer Wavelet Transform (IWT), and the Discrete Cosine Transform (DCT). JPEG, a standard image compression technique employs DCT. An example of utilizing DWT is the employment of an adaptive data embedding technique by using OPAP in which the secret data is hidden in the Integer Wavelet coefficients of the cover image [7]. This will maximize the hiding capacity as much as possible by overcoming the problems of complex computations and distortions. A pseudo-random generator function is used to select different embedding positions along with a Kohonen neural network, in which pixels are classified into different classes of sensitivity [8]. Kohonen neural networks are used because they are a relatively simple network to construct that can be trained very rapidly. Thus the adaptive data hiding function along with Kohonen network increases the system security.

The rest of the paper is organized as follows: Section II provides a brief prefatory to Integer wavelet transform and Kohonen neural network. In Section III the proposed system is presented. Experimental results are illustrated in Section IV, prior to Conclusions in Section V.

\section{THE STEGANOGRAPHY METHOD}

\subsection{Integer Wavelet Transform (IWT)}

In recent years, there has been a growing interest in Integer Wavelet Transforms for image processing applications. Such transforms are invertible in finite-precision arithmetic (i.e., reversible), map integers to integers, and approximate linear wavelet transforms. Due largely to these properties, Integer wavelet transforms are extremely useful for image compression systems requiring efficient handling of lossless coding, minimal memory usage, or low computational complexity, lossy-to-lossless recovery of images. Integer wavelet transform maps an integer data set into another integer data set. Integer wavelet transform (IWT) has the important property that IWT coefficients have the same dynamical range as the original signal. In discrete wavelet 
transform, the wavelet filters employed have floating point coefficients and any truncations of the floating point values of the pixels may lead to loss of hidden data. To avoid problems with floating point precision of the wavelet filters, IWT is used and hence there will be no loss of information through forward and inverse transform [9]. The LL subband of the original image (lena) in the case IWT is found to be a secure replica with smaller scale of original image whereas the resulting LL subband is somewhat distorted (as shown in Fig $1[2])$ in the case of DWT.

In many applications (e.g. multimedia) the input data consist of integer samples. Fortunately the lifting scheme can be modified easily to a transform [10] that maps integers to integers and that is still reversible. This is done by adding some rounding operations (indicated by curly braces), at the expense of introducing a non-linearity in the transform. $\gamma \mathrm{j} \quad \gamma \mathrm{j}-\{\mathrm{P}(\lambda \mathrm{j})\}$,

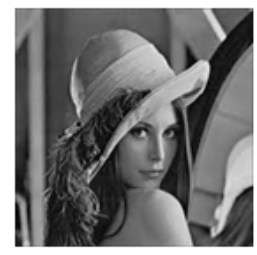

$\lambda \mathrm{j} \quad \lambda \mathrm{j}+\{\mathrm{U}(\gamma \mathrm{j})\}$

(1)

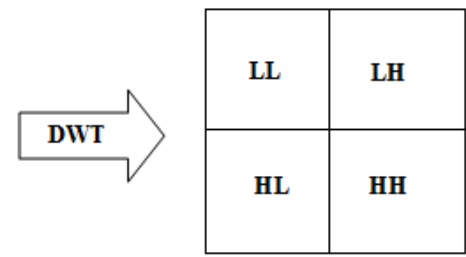

(a)

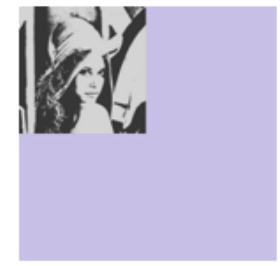

(b)

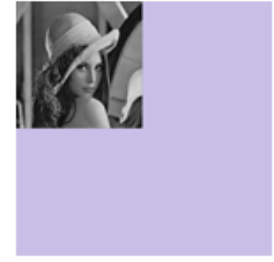

(c)
Fig. 1 (a) Original image Lena and its analysis in wavelet domain. (b) One level 2DDWT in subband LL. (c) One level 2DIWT in subbband $L L$.

The Haar wavelet transform using lifting scheme can be written as follows:

$$
\begin{aligned}
& d_{l, n}=s_{0,2 n+1}-s_{0,2 n} \\
& s_{l, n}=s_{0,2 n}+d_{l, n / 2}
\end{aligned}
$$

The Haar wavelet transform can be written as pairwise averages and differences

$$
\begin{aligned}
& s_{1, n}=\left(s_{0,2 n}+s_{0,2 n+1}\right) / 2 \\
& d_{1, n}=s_{0,2 n+1}-s_{0,2 n}
\end{aligned}
$$

From (1) and (2) the integer wavelet transform can be designed as below:

$$
\begin{aligned}
& d_{1, n}=s_{0,2 n+1}-s_{0,2 n} \\
& s_{l, n}=s_{0,2 n}+\left\lfloor d_{1, n / 2}\right\rfloor
\end{aligned}
$$

The inverse transform is given by

$$
\begin{aligned}
& s_{0,2 n}=s_{1, n}-\left\lfloor d_{l, n / 2}\right\rfloor \\
& s_{0,2 n+1}=d_{1, n}+s_{0,2 n}
\end{aligned}
$$

\subsection{Kohonen Neural Network (KNN)}

The SOM (Self Organizing Map) [11] introduced by Kohonen is an unsupervised learning method which has both clustering and visualization properties and creates a correspondence between the input space of stimuli and the output space constituted of the code words or neurons. The learning algorithm ensures that the most highly activated node as well as its neighbors move towards a sample presented to the network. The networks are self organizing in such a way that nodes tend to attain weight vectors that capture characteristics of the input vector space, with the neighborhood relation translating into proximity in Euclidean space, even if the initial values of weight vectors are arbitrary.

The Kohonen neural network contains only an input and output layer of neurons. There is no hidden layer in this network. When a training example is fed to the network, its Euclidean distance to all weight vectors is calculated. The neuron with weight vector most similar to the input is called the best matching unit (BMU). The weights of the BMU and neurons close to it in the Self Organising Lattice (SOM) lattice are adjusted towards the input vector. The magnitude of the change decreases with time and with distance from the BMU. The update formula for a neuron with weight vector $\mathrm{Wv}(\mathrm{t})$ is.

$$
\mathrm{Wv}(\mathrm{t}+1)=\mathrm{Wv}(\mathrm{t})+\Theta(\mathrm{v}, \mathrm{t}) \alpha(\mathrm{t})(\mathrm{D}(\mathrm{t})-\mathrm{Wv}(\mathrm{t}))
$$

where $\alpha(t)$ is a monotonically decreasing learning coefficient and $D(t)$ is the input vector. The neighborhood function $\Theta(v$, t) depends on the lattice distance.

In the proposed method [8] depending on the absolute sum of wavelet contrast coefficients, Kohonen network is trained. This method checks all pixels present in cover image and calculates the degree of sensitivity of pixels with trained neural network so that pixels can be classified into different classes of sensitivity. Pixels in less sensitive areas can carry more hidden data as compare to those which are in high sensitive area of human vision. According to the contrast sensitivity, the trained network classifies the blocks of cover image into 3 categories. Blocks with largest sensitivity is one class A1, while that with smallest is another class $\mathrm{A} 2$, and rest is the third class A3. Then the secret information is embedded in the DWT coefficients.

\section{PROPOSED METHOD}

The proposed method uses an adaptive data hiding scheme along with Kohonen neural network. During the embedding process, all pixels present in the cover image are evaluated and the degree of sensitivity of pixels is calculated so that pixels can be classified into different classes of sensitivity. Depending on the sensitivity the wavelet coefficients are classified. Each of the selected coefficients hides different number of secret message bits according to the adaptive data hiding capacity function. After embedding the data OPAP is done. This will reduce the data insertion error.

\subsection{Embedding Algorithm}

The embedding process which is shown in Fig 2 is explained as below:

Step 1: Take a $512 \times 512$ image as the input cover image.

Step 2: Apply histogram modification to prevent the overflow or underflow, which occurs when the stego-image pixel values are not in the range (0-255) [12]. 
Step 3: Divide the input image into $8 \times 8$ blocks.

Step 4: Apply the Integer Wavelet Transform to each block using 2D Haar IWT [12].

Step 5: Calculate the absolute sum of wavelet contrast coefficients $C$. The smaller the value of $C$ is, the lesser the secret information is.

Step 6: Train the Kohonen network according to the calculated coefficient C. A threshold is used to classify the blocks into 3 categories. Blocks with largest $\mathrm{C}$ is a class $\mathrm{A} 1$, while that with smallest is a class A2, and rest is A3.

Step 7: Calculate the number of message bits to be used in each of the wavelet coefficient. The length of LSBs (L) is calculated as below [12].

$$
L=\left\{\begin{array}{l}
K+3, \text { if } C \geq 2 K+3 \\
K+2, \text { if } 2 K+2 \leq C<2 K+3 \\
K+1, \text { if } 2 K+1 \leq C<2 K+2 \\
K, \text { if } C<2 K+1,
\end{array}\right.
$$

$\mathrm{K}=1,3,4$ (from experiments of different values of $\mathrm{K}$ we divided the system into 3 cases of operation depending on the requirements of the user). $\mathrm{C}$ is the absolute wavelet coefficient value and $K$ is the minimum length to be used in each coefficient.

Step 8: Replace the L LSBs of randomly chosen coefficients with $\mathrm{L}$ bits of secret data. This is done by means of a secret key, which is an $8 \times 8$ matrix. The secret key consists of only 1 's and 0 's.

Step 9: Apply the Optimal Pixel Adjustment Process to reduce the error difference between the original coefficient value and modified coefficient value. The main idea of using the optimum pixel adjustment (OP A) algorithm is to minimize the error difference between the original coefficient value and the altered value. This is done by checking the right next bit to the modified LSBs to make their difference minimum. The algorithm used in [13] depends on the difference $(\delta \mathrm{i})$ between original value $\mathrm{P}(\mathrm{x}, \mathrm{y})$ and the modified value $\mathrm{P}^{\prime}(\mathrm{x}, \mathrm{y})$.

$$
\delta \mathrm{i}=\mathrm{Pi}^{\prime}(\mathrm{x}, \mathrm{y})-\mathrm{Pi}(\mathrm{x}, \mathrm{y})
$$

The OPA process modifies the altered value in the following way:

$$
\begin{aligned}
& \text { Case } 1(-2 \mathrm{~K}<\delta \mathrm{i}<-2 \mathrm{~K}-1) \\
& \text { If } \mathrm{Pi}^{\prime}(\mathrm{x}, \mathrm{y})<256-2 \mathrm{~K} \\
& \mathrm{Pi}^{\prime}(\mathrm{x}, \mathrm{y})^{*}=\mathrm{Pi}^{\prime}(\mathrm{x}, \mathrm{y})+2 \mathrm{~K} \\
& \text { Else } \mathrm{Pi}^{\prime}(\mathrm{x}, \mathrm{y})^{*}=\mathrm{Pi}^{\prime}(\mathrm{x}, \mathrm{y}) \\
& \mathrm{Pi}^{\prime}(\mathrm{x}, \mathrm{y})^{*}=\mathrm{Pi}^{\prime}(\mathrm{x}, \mathrm{y})
\end{aligned}
$$

Step 10: Obtain the Stego image by calculating the inverse IWT of each 8x8 modified block.

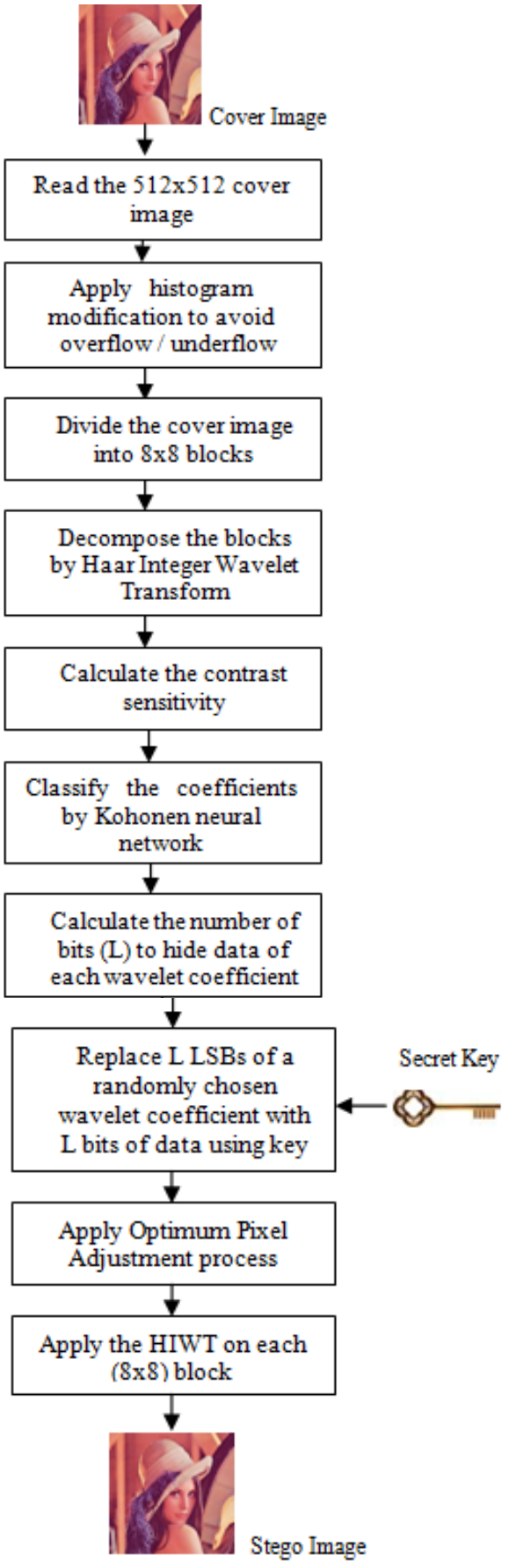

Fig. 2 The Embedding Process

\subsection{Extraction Algorithm}

The extraction process is as shown in Fig 3. It is a simple process and does not require the cover image to recover the secret data.

Step 1: Take the 512x512 stego image.

Step 2: Divide the input image into $8 \times 8$ blocks.

Step 3: Apply the Integer Wavelet Transform to each block using 2D Haar IWT.

Step 4: Classify the coefficients by Kohonen network.

Step 5: Calculate the number of message bits to be used in each of the wavelet coefficient. 
Step 6: Use the secret key to generate the selected coefficients to embed secret data.

Step 7: Extract L bits from each selected coefficient.

Step 8: Gather all the extracted bits to generate the secret message.

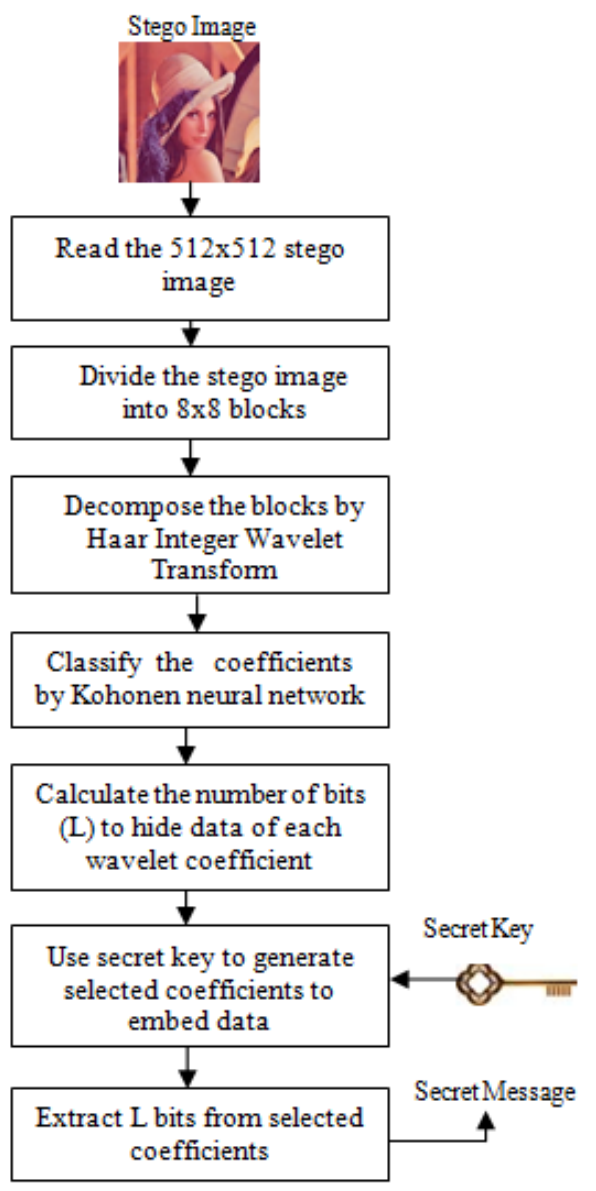

Fig. 3 The Extraction Process

\section{IMPLEMENTATION AND RESULT ANALYSIS}

The proposed method is applied on $512 \times 512$ colour images such as Lena, Baboon and Barbara. The simulation is implemented in Matlab 7 or above (eg. Matlab 7.1 and Matlab 7.9). Fig 4 shows the original cover images and their corresponding Integer Wavelet Transforms. Fig 5 gives the stego images which are obtained by the proposed method after embedding a data text.

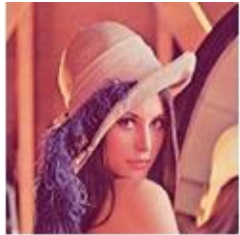

(a) Lena

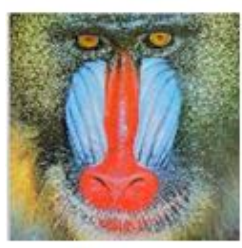

(c) Baboon

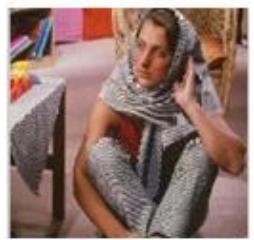

(e) Barbara

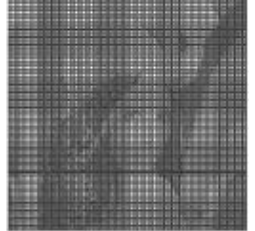

(b)

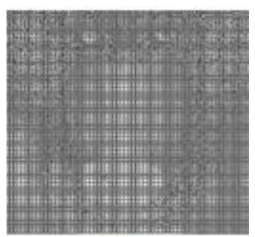

(d)

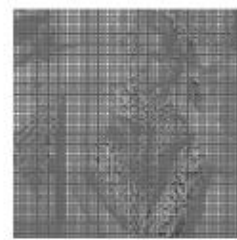

Fig. 4 Original images and their corresponding IWT

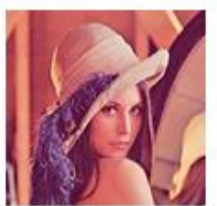

(a)
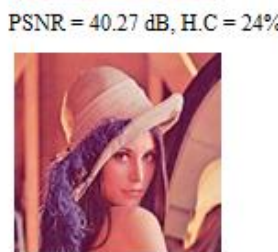

(b) $\mathrm{PSNR}=39.72 \mathrm{~dB}, \mathrm{H} \cdot \mathrm{C}=30 \%$

Fig 5. Output stego images with PSNR and H.C

\subsection{Peak Signal to Noise Ratio (PSNR):}

The PSNR measures how much difference has occurred to the original image by the process of embedding data in the input cover image. Thus the quality of stego image can be estimated. The PSNR is calculated by (10)

$$
\mathrm{PSNR}=10 \log 10\left(255^{2} / \mathrm{MSE}\right)
$$

Where

$$
\operatorname{MSE}=(1 / \mathrm{MxN}) \sum \mathrm{x}=1 \quad \sum \mathrm{y}=1\left(\mathrm{P}(\mathrm{x}, \mathrm{y})-\left(\mathrm{P}^{\prime}(\mathrm{x}, \mathrm{y})\right)^{2}\right.
$$

The MSE is the Mean Square Error, $\mathrm{P}(\mathrm{x}, \mathrm{y})$ is the input cover image and $\mathrm{P}^{\prime}(\mathrm{x}, \mathrm{y})$ is the output image. $\mathrm{M}$ and $\mathrm{N}$ are the size of the input image. A higher PSNR (ref. to table I) value implies better image quality and lower distortion in the stego image.

\subsection{Hiding Capacity (H.C):}

Fundamentally, data hiding capacity of a steganographic scheme can be defined as the amount of information it can hide within the cover image. As with any method of storing data, this can be expressed as a number of bits, which indicates the maximum message size that might be inserted 
into an image. The hiding Capacity $\mathrm{C}$ is measured as bits per pixel (bpp) which is calculated as follows:

$$
\mathrm{H} \cdot \mathrm{C}=(\mathrm{B} / \mathrm{MxN}) \text { bpp }
$$

where $\mathrm{M}$ and $\mathrm{N}$ are the size of the input image and $\mathrm{B}$ is the size of the secret data embedded.

Table 1. Comparison showing the results of various methods

\begin{tabular}{|l|l|c|}
\hline Image & Method Used & PSNR(dB) \\
\hline Lena & Proposed method & 40.27 \\
\hline Baboon & Proposed method & 32 \\
\hline Barbara & Proposed method & 32.35 \\
\hline Lena & $\begin{array}{l}\text { AdaptiveTechnique } \\
\text { using IWT }\end{array}$ & 31.8 \\
\hline Baboon & $\begin{array}{l}\text { AdaptiveTechnique } \\
\text { using IWT }\end{array}$ & 30.89 \\
\hline Barbara & $\begin{array}{l}\text { AdaptiveTechnique } \\
\text { using IWT }\end{array}$ & 31.35 \\
\hline Lena & DCT & $\mathbf{3 1 . 1 8 7 5}$ \\
\hline Baboon & DCT & 26.2159 \\
\hline & DCT & \\
\hline & & \\
\hline
\end{tabular}

\section{CONCLUSION}

This paper presented a novel data hiding technique which hides the secret data by combining integer wavelet transform and the optimum pixel adjustment algorithm to maximize the hiding capacity of the system compared to other systems. The proposed system embeds secret information using a secret key only known to both sender and receiver. This is done by means of a stego key, which is an $8 \times 8$ matrix. The secret key consists of only 1 's and 0 's. Thus each time it is easy to alter the key and make the system more preventive against any attacks. Also this is an adaptive system which embeds different number of bits in each wavelet coefficient, whose data embedding capacity is calculated using a Kohonen neural network in order to increase the hiding capacity without sacrificing the visual quality of resulting stego image. The proposed system also reduces the difference between original coefficients values and modified values by using the optimum pixel adjustment algorithm.

\section{REFERENCES}

[1] R.J. Anderson, M.G. Khun, "Information hiding- A survey", Proceedings of IEEE, Vol. 87, 1999

[2] R.O. El Safy, H.H. Zayed, A. El Dessouki, "An Adaptive Steganographic Technique Based on Integer Wavelet Transform", International Conference on Networking and Media Convergence, 2009.

[3] H.W. Tseng and C.C. Chnag, "High capacity data hiding in jpeg compressed images”, Informatica, Vol. 15, 2004.

[4] ] C.K.Chan and L.M. Chang, "Hiding data in images by simple LSB substitution", Pattern Recognition, pp.469-474, March 2004

[5] N.Wu and M.Hwang, "Data hiding: current status and key issues", International Journal of Network Security, Vol. 14, pp. 1-9, Jan 2007.

[6] W.Chen, “ A comparative study of information hiding schemes using amplitude, frequency and phase embedding", PhD thesis, National Cheng Kung University, Taiwan, May 2003.

[7] Elham Ghasemi, Jamshid Shanbehzadeh, Bahram Zahir Azami, "A steganographic method based on Integer wavelet transform and genetic algorithm", International Conference on Communications and Signal Processing, 2011.

[8] Arun Rana,Nitin Sharma, Amandeep Kaur, "Image steganogrphy based on Kohonen neural network", International Journal of Engineering Research and Applications (IJERA), Vol. 2, Issue 3, May-Jun 2012, pp.2234-2236.

[9] Michael David Adams, "Reversible Integer-to-Integer Wavelet Transforms for Image Coding", $\mathrm{PhD}$ thesis, University of British Columbia, Sep 2002.

[10] Geert Uytterhoeven, Dirk Roose, Adhemar Bultheel, "Integer Wavelet Transforms using the Lifting Scheme", Department of Computer Science, International Conference on Communication systems and Computers, Vol. 1, pp. 6251-6253.

[11] Kohonen. T, "Self-Organizing and Associative Memory", Springer-Verlag,Heidelberg.

[12] B.Lai and L.Chang, "Adaptive Data Hiding for Images based Haar Discrete Wavelet Transform", Lecture noted in Computer Science, Vol. 4319/2006.

[13] H.H. Zayed, "A High-Hiding Capacity Technique for Hiding Data in Images based on K-bit LSB substitution", International Conference on Artificial Intelligence Applications, Cairo, Feb. 2005. 